\title{
Validation of the registered underlying causes of stillbirth
}

\author{
L EDOUARD
}

From the Department of Research and Services in Education, The Middlesex Hospital Medical School, University of London, London WIP 7PN

SUMmaRY Clinical case notes were used to validate the registered causes of 200 stillbirths. The diagnoses were concordant in 69\% of cases using a 14-category classification of fetal death, discrepancies being mainly due to available information not being used in the assignment of cause of stillbirth. Excluding cases of congenital malformation, 34\% of stillbirths occurred in labour.

Although the main purpose of death certification is sociolegal, a comprehensive statement of cause of death with specific diagnoses in their correct sequence fulfils the requirements of an epidemiological data bank. ${ }^{1}$ The World Health Organisation $^{2}$ emphasised the importance of studying the quality of perinatal mortality statistics in the context of technological advances in the field of data processing.

Fedrick and Butle $r^{3}$ found wide discrepancies in the codings for pulmonary lesions between the recorded causes of 1656 neonatal deaths in the British Perinatal Mortality Survey of 1958 and those registered on the death certificates. Clarke and Whitfield $d^{4}$ examined the hospital notes of all deaths in England and Wales in 1977 where haemolytic disease of the newborn had been registered as a cause, whether principal, antecedent, or contributory. About a quarter of these deaths seem unrelated to haemolytic disease of any type: hydrops, without mention of cause, has been coded as haemolytic disease, and stillbirths of unknown aetiology were often attributed to rhesus haemolytic disease when the mother was rhesus negative. That source of inaccuracy was arithmetically balanced out by cases in which haemolytic disease of the newborn had not been selected by the coders as the underlying cause. The value of these findings, however, was limited as the study excluded cases where haemolytic disease had not been entered on the certificate despite being the cause of death.
The aim of the current study was to determine their accuracy by using data from clinical case records.

\section{Method}

Stillbirths occurring during 1973 and 1977 in eight maternity units of the North-west Thames Health Region were studied, the Office of Population Censuses and Surveys (OPCS) supplying the registered underlying cause of each stillbirth.

I personally examined the clinical case notes to obtain data on the age, parity, and smoking habit of the mother, gestational ages at booking and at fetal death, complications of pregnancy (multiple pregnancy, pre-eclampsia, antepartum haemorrhage, and intrauterine growth retardation), procedures in labour (induction or acceleration of labour and mode of delivery), the time of fetal death related to labour, sex, and birthweight of the fetus, and, finally, performance of a necropsy.

I reconsidered the cause of each stillbirth using all available information from the case notes but without the knowledge of the registered cause compiled by OPCS, which was linked to the rest of the data at the end of the survey. ${ }^{5}$ Initially coded into the 100 rubrics of the P list of the International Classification of Diseases, these causes were reclassified into 14 broader but clinically more meaningful categories previously used in a national study of perinatal mortality. All data were entered directly on to specially designed mark sense computer $\operatorname{cards}^{7}$ to 
ensure confidentiality and to simplify handling of data.

The ratio of reconsidered cases to registered cases was calculated for each category of fetal wastage.

\section{Results}

A total of 218 cases identified from the list supplied by OPCS were included in the study. The case notes showed that three cases had been wrongly registered as stillbirths: two early neonatal deaths had been registered as due to difficult labour and placental haemorrhage, and a spontaneous abortion had been registered as due to prematurity.

Clinical notes were missing in 15 cases, registered causes being in the following categories: umbilical cord complication (three cases), congenital malformation, anoxia of unspecified cause, and pre-eclampsia syndrome (two each), prematurity, multiple pregnancy, placental haemorrhage, placental insufficiency, medical condition of mother, and miscellaneous (one each). The survey is therefore based on data from 200 case notes.

Data sought were available in almost all cases. Relevant information was missing from more than two cases for the following variables only: (1) the birthweight was missing in five cases $(2.5 \%)$; (2) the gestational age at booking was unavailable in seven cases (3.5\%): the usual reason was a transfer from the intended place of confinement when complications occurred; and (3) the smoking habit was missing in 103 cases $(51 \cdot 5 \%)$. A necropsy was performed in 134 cases $(67 \%)$.

The registered and reconsidered diagnoses were concordant for 137 cases $(68.5 \%)$ in terms of the 14-category classification. For each category of stillbirth, the appropriateness of the diagnosis was investigated by a $2 \times 2$ contingency table tabulating registered cause as recorded by OPCS and the reconsidered cause as judged from examination of the clinical notes.

The extent of agreement between registered and reconsidered causes for each of the 14 categories of fetal death are summarised in the table, where the columns represent cases with (a) both causes in that category, (b) the registered cause but not the reconsidered cause in that category, and (c) the reconsidered cause but not the registered cause in that category. The correction factor to be applied to the number of registered causes for each category was calculated as the total number of reconsidered cases $(a+c)$ expressed as a proportion of the total number of registered cases $(a+b)$. A correction factor of unity means that no correction is necessary; if greater than one, more cases should have been registered under that category and if less than one fewer cases should have been registered as such.

Congenital malformation represents a highly reliable diagnostic category: it was the reconsidered cause of 37 stillbirths compared with being the registered cause of 35 stillbirths, diagnoses being concordant in 33 of these cases-the number of registered cases should therefore be corrected by a multiplying factor of 1.1 (table). A diagnosis of congenital malformation was reliable both in the individual case and at the population level, whereas that of pre-eclampsia as an underlying cause of stillbirth was inaccurate at individual patient level but reliable at population level. The two assessments agreed with a diagnosis of pre-eclampsia in 10 cases but disagreed in a further 10 cases (table): pre-eclampsia was the registered cause in four cases

Validity of registered causes of stillbirth

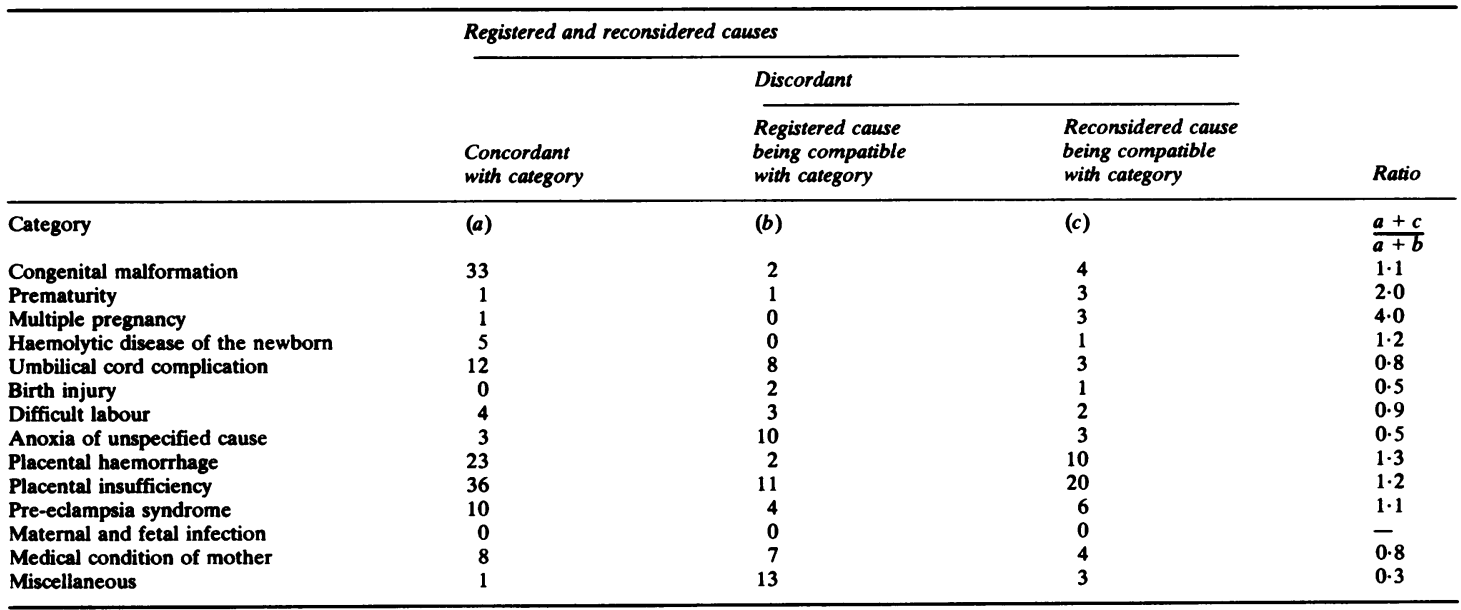


and the reconsidered cause in the other six. Hence pre-eclampsia was the reconsidered cause of 16 stillbirths compared with 14 from the registration process - the correction factor was close to unity as the false-positives balanced the false-negatives.

Correction factors greater than unity assigned to placental haemorrhage and placental insufficiency indicate a deficiency of cases ascribed to these categories in the registration process as compared with the examination of clinical notes in this study. Ten of the cases ascribed to placental haemorrhage in this study had been registered under the following categories: placental insufficiency (two cases) pre-eclampsia (four), medical condition of mother (one), and miscellaneous (three). On the other hand, two cases registered as placental haemorrhage were thought to be due to difficult labour and the miscellaneous category.

The deficiency in cases registered as placental insufficiency was accounted for mainly through using the following categories at registration: anoxia of unspecified cause (six cases), miscellaneous (six), and umbilical cord complication (five). Five of these six cases registered in the category of anoxia of unspecified cause were macerated at birth, whereas all six cases in the miscellaneous category had been registered with vague diagnoses such as intrauterine death. Those five cases registered as umbilical cord complication had irrelevant conditions of the cord, three of them having previously shown clinical features of intrauterine growth retardation.

Eleven cases registered as placental insufficiency were redistributed as follows: two each to pre-eclampsia, placental haemorrhage, medical condition of mother, and multiple pregnancy, and one each to haemolytic disease of the newborn, umbilical cord complication, and anoxia of unspecified cause. Hence the term placental insufficiency was often used as an underlying cause at registration of stillbirth when a more specific diagnosis was possible on clinical grounds.

The category of anoxia of unspecified cause had a correction factor of $0 \cdot 5$. The diagnoses were concordant in only three cases and 10 cases registered under that category were reallocated to other categories: fetal death had occurred before the onset of labour in six cases, and there was a more specific underlying condition in the other four cases. Hence use of the term anoxia was not restricted to the certification of intrapartum stillbirths with no apparent underlying cause clinically. These 10 cases were reassigned to placental insufficiency (six), umbilical cord complication, birth injury, medical condition of mother, and miscellaneous (one each).

Of the cases registered in the miscellaneous category, 13 were reallocated: placental insufficiency (six cases), congenital malformation and placental haemorrhage (three each), and prematurity (one).

Excluding the 37 cases of congenital malformation, there were 56 intrapartum stillbirths distributed as follows: placental haemorrhage (17), umbilical cord complication (eight), difficult labour and anoxia of unspecified cause (six each), placental insufficiency (five), pre-eclampsia (four), medical condition of mother and prematurity (three each), miscellaneous (two), birth injury and multiple pregnancy (one each).

\section{Discussion}

Although obstetric enigmas can be investigated by retrospective analysis of clinical records ${ }^{8}$ and prospective regional surveys, ${ }^{9}$ the value of national repositories of routine statistics should not be underestimated. ${ }^{10}$ Their limitations, however, include the degree of accuracy of diagnoses. Registered causes of death have been validated by either extracting the principal condition stated on the clinical summary sheet, ${ }^{11}$ or using diagnostic information previously collected for a different purpose $^{3}$ or doing postal surveys. ${ }^{4}$ The potential of case note inspection should be exploited by further regional studies as their feasibility has been shown.

The structured case notes widely used in obstetric practice ensures that standard data are usually available. The few cases of missing birthweight in well-structured case notes tended to be associated with a total disregard for information requested on a separate neonatal record sheet. Other cases of missing data were often associated with a transfer of the mother to another institution when complications occurred.

As information on death certificates can bear little relation to the preceding events, some think that these certificates are not worth the paper they are written on. The retrospective validation of the registered causes of stillbirth depends heavily on the information available in the notes. When registered and reconsidered causes are discordant, the former could be the correct one when essential information is missing from the notes. Even with complete records, discordant diagnoses can result from genuine differences in either clinical opinion or use of terms such as placental insufficiency. Discordance may also be due to different attitudes towards classification $^{12}$ : my approach, which concentrated on the $P$ list, differs from those of the clinician who ignores statistical classifications and the national coding clerk who adheres to the four-digit categories of the International Classification of Diseases. Commenting on these problems, Greenwood ${ }^{13}$ stated that, "making the best the enemy of the good is a sure 
way to hinder any statistical progress. The scientific purist, who will wait for medical statistics until they are nosologically exact, is no wiser than Horace's rustic waiting for the river to flow away."

These limitations, however, should not divert attention away from actual deficiencies in the certification of cause of stillbirth. Although it seems desirable to perform necropsies to obtain more information on cause of perinatal death, ${ }^{2}$ it is as important to consider ways of using available information. Correct completion of the death certificate should be stressed to practitioners, who must also be made aware of the use of the information collected. Better co-operation between pathologist and clinician is needed to ensure elucidation of underlying conditions and preventable causes.

Although it is desirable to use routinely collected statistics for monitoring the effect of changes in clinical practice on fetal outcome, it is difficult to interpret national secular trends for most of the registered causes of perinatal mortality in the absence of validation studies..$^{5}$ An extension of the current study would allow the evaluation of recent changes in certification practice. The greater number of cases in an extended series would also enable the identification of high risk factors associated with conditions such as anoxia of unspecified cause, which may not be clinically evident until imminent fetal death and hence more likely to benefit from continuous intrapartum monitoring. The demonstration of the relative importance of intrapartum stillbirth lends support to emphasis on labour in a risk approach to perinatal care.

Effective health service management depends heavily on assessment of need for services, use of resources, and outcome of treatment; the quality of relevant information can be greatly improved through better use of readily available data. The truth that has ensued from this study may be uncomfortable to epidemiologists, obstetricians, and consumers. It has, however, unveiled shortcomings which, if remedied, should lead to a better health information system for the ultimate benefit of everyone.
I thank Professor Eva Alberman and Professor R W Beard for their helpful suggestions and the North-west Thames Regional Health Authority for support.

Requests for reprints to Dr L Edouard, Department of Research and Services in Education, The Middlesex Hospital Medical School, Cleveland Street, London W1P 7PN.

\section{References}

${ }^{1}$ Adelstein AM. Certifying cause of death. Health Trends 1977; 9: 78-81.

${ }^{2}$ World Health Organisation. Social and biological effects on perinatal mortality. Report on an international comparative study. Vol 1. Budapest: Statistical Publishing House, 1978.

${ }^{3}$ Fedrick J, Butler NR. Accuracy of registered causes of neonatal deaths in 1958. Br J Prev Soc Med 1972; 26: 101-5.

'Clarke C, Whitfield AGW. Deaths from rhesus haemolytic disease in England and Wales in 1977: accuracy of records and assessment of anti-D prophylaxis. $\mathrm{Br} \mathrm{Med} J$ 1979; i: 1665-9.

${ }^{5}$ Edouard L. Trends in fetal outcome. London: University of London, 1980. (MSc (social medicine) thesis.)

- Edouard L, Alberman E. National trends in the certified causes of perinatal mortality, 1968 to $1978 . \mathrm{Br} J$ Obstet Gynaecol 1980; 87: 833-8.

${ }^{7}$ Edouard LJ, Harris FTC. A computer card for marking by the examiner. British Journal of Educational Technology 1976; 3: 37-40.

${ }^{8}$ Baird D. Social factors in obstetrics. Lancet 1949; i: 1079-83.

${ }^{9}$ Chalmers I. Perinatal epidemiology: strengths, limitations and possible hazards. In: Beard RW, Campbell S, eds The current status of fetal heart rate monitoring and ultrasound in obstetrics. London: Royal College of Obstetrics and Gynaecologists, 1978: 12-25.

${ }^{10}$ Farr W. Letter to the Registrar-General from William Farr Esq. In: First annual report of the Registrar-General of births, deaths and marriages in England. Appendix P. London: HMSO, 1839: 63-81.

${ }^{11}$ Alderson MR, Meade TW. Accuracy of diagnosis on death certificates compared with that in hospital records. Br J Prev Soc Med 1967; 21: 22-9.

${ }^{12}$ Cochrane AL, Moore F. Death certification from the epidemiological point of view. Lancet 1981; ii: 742-3.

${ }^{13}$ Greenwood M. Medical statistics from Graunt to Farr-The Fitzpatrick Lectures for the years 1941 and 1943, delivered at the Royal College of Physicians of London in February 1943. Cambridge: University Press, 1948: 28. 